\title{
Avaliação de escore de condição corporal e famacha em ovelhas suffolk a pasto
}

\author{
Avaliation of the body condition score and famacha in suffolk ewes based pasture system \\ Evaluación del grado de condición corporal y famacha en ovejas suffolk en sistema de pastoreo
}

Recebido: 18/01/2022 | Revisado: 26/01/2022 | Aceito: 27/01/2022 | Publicado: 29/01/2022

Thaís Campos de Freitas

ORCID: https://orcid.org/0000-0003-3942-599X Instituto Federal de Educação, Ciência e Tecnologia do Rio Grande do Sul, Brasil E-mail: thaiscamposdefreitas@gmail.com

Ana Lúcia Barreto da Costa

ORCID: https://orcid.org/0000-0002-0807-2581

Instituto Federal de Educação, Ciência e Tecnologia do Rio Grande do Sul, Brasil

E-mail: ad.costa1998@gmail.com

Edriane Mara Sbardelotto

ORCID: https://orcid.org/0000-0003-2985-5996 Instituto Federal de Educação, Ciência e Tecnologia do Rio Grande do Sul, Brasil E-mail: edrianesbardelotto@gmail.com

Elísio de Camargo Debortoli

ORCID: https://orcid.org/0000-0001-5594-2810 Instituto Federal de Educação, Ciência e Tecnologia do Rio Grande do Sul, Brasil E-mail: elisio.debortoli@sertao.ifrs.edu.br

Juliano Hideo Hashimoto

ORCID: https://orcid.org/0000-0003-3659-5635 Instituto Federal de Educação, Ciência e Tecnologia do Rio Grande do Sul, Brasil E-mail: juliano.hashimoto@sertao.ifrs.edu.br

Jamile Cristina Deola Sada

ORCIDI: https://orcid.org/0000-0002-8096-8665 Instituto Federal de Educação, Ciência e Tecnologia do Rio Grande do Sul, Brasil E-mail: jamile.sada@sertao.ifrs.edu.br Joilson Gradin

ORCID: https://orcid.org/0000-0002-0930-3468 Instituto Federal de Educação, Ciência e Tecnologia do Rio Grande do Sul, Brasil E-mail: jilson.gradin@sertao.ifrs.edu.br

\begin{abstract}
Resumo
$\mathrm{Na}$ ovinocultura, uma das maiores perdas econômicas é a verminose, desta forma, o manejo integrado pode promover um bom desempenho na produção e consequentemente gerar menos perdas. Este trabalho teve por objetivo avaliar o perfil sanitário, por meio da associação entre a aplicação do método FAMACHA e a avaliação do Escore de Condição Corporal (ECC), de ovinos da raça Suffolk no IFRS - Campus Sertão. A amostra foi composta por 27 animais adultos totalizando 725 avaliações no ano de 2019. Foi observado que o método FAMACHA proporcionou redução nos gastos com vermífugos em 46,3\% quando comparado ao manejo tradicional com duas vermifugações em todo o rebanho no período de análise. Como é um tratamento seletivo, associando as informações do ECC com o grau FAMACHA, apenas $3,72 \%$ dos animais foram vermifugados enquanto que os outros $96,28 \%$ possuíam carga parasitária baixa ou conseguiram suportar a carga parasitária sem apresentar sinais clínicos. Também foi possível classificar o rebanho em animais resistentes, resilientes e suscetíveis para a verminose. Tais resultados demonstram a importância do controle zootécnico e acompanhamento contínuo do rebanho, buscando proporcionar melhor desempenho produtivo e sanitário e consequentemente evitando o uso indiscriminado de antiparasitários e aprimorando a seleção de animais.
\end{abstract}

Palavras-chave: Controle zootécnico; Manejo sanitário; Verminose.

\begin{abstract}
It is known that the cause of the greatest economic losses is worms in sheep production, in this way, integrated management can promote good performance in production and consequently generate less losses. Thus, the integrated handle can promote a good performance in production and consequently reduce the damages. This study aimed to evaluate the sanitary profile, through the association between the application of the FAMACHA method and the evaluation of the Body Condition Score (BCS), of Suffolk breed sheep in IFRS - Campus Sertão. The flock of 27 adult sheep were followed, totaling 725 evaluations in the year 2019. The FAMACHA method provided the reduction in expenses with anthelmintic drugs by $46.3 \%$ when compared to the traditional handle with two applications of anthelmintic drugs in sheep in the period analyzed. In the selective treatment, associated with BCS information and FAMACHA score, only $3.72 \%$ of the animals received anthelmintic drugs, while most of the flock with relatively few parasites or were able to support the parasite load without showing clinical signs. With the results, these sheep were
\end{abstract}


classified into three groups into resistant, resilient and susceptible to verminosis. The study demonstrated the importance of zootechnical control and continuous monitoring of the flock, seeking to provide better productive and sanitary performance and consequently avoiding the indiscriminate use of anthelmintic drugs and improving the selection of animals.

Keywords: Zootechnical control; Sanitary handle; Verminosis.

\section{Resumen}

En la ganadería ovina, una de las mayores pérdidas económicas son las parasitosis, de esta forma, el manejo integrado puede promover un buen desempeño en la producción y consecuentemente generar menos pérdidas. Por lo tanto, la gestión integrada puede promover un buen desempeño en la producción y, en consecuencia, generar menos pérdidas. Este estudio tuvo como objetivo evaluar el perfil de salud, a través de la asociación entre la aplicación del método FAMACHA y la evaluación del Grado de condición corporal (GCC), de ovejas Suffolk en IFRS - Campus Sertão. La muestra estuvo conformada por 27 animales adultos totalizando 725 evaluaciones en el año 2019. Se observó que el método FAMACHA proporcionó una reducción del 46,3\% en el gasto en desparasitaciones en el rebaño en comparación con el manejo tradicional con dos desparasitaciones en todo el rebaño en el periodo de análisis. Al tratarse de un tratamiento selectivo, asociando la información de GCC con el grado de FAMACHA, solo el 3,72\% de los animales estaban desparasitados mientras que el 96,28\% restante tenían carga parasitaria baja o podían soportar la carga parasitaria sin presentar signos clínicos. También fue posible clasificar el rebaño en animales resistentes, resilientes y susceptibles a los gusanos. Estos resultados demuestran la importancia del control zootécnico y el seguimiento continuo del rebaño, buscando brindar un mejor desempeño productivo y sanitario y consecuentemente evitando el uso indiscriminado de antiparasitarios y mejorando la selección de animales.

Palabras clave: Control zootécnico; Gestión sanitaria; Gusanos.

\section{Introdução}

A raça Suffolk tem origem dos condados de Norfolk, Cambridge, Essex e Suffolk, no sudoeste da Inglaterra, sendo formada a partir dos cruzamentos entre as raças Southodown com Norfolk, resultando em um ovino com grande desenvolvimento corporal, robusto, prolífico, com a conformação tipicamente carniceira (ARCO, 2021).

No Rio Grande do Sul, a ovinocultura ainda é explorada em sua grande maioria de forma extensiva, tendo sua produção conciliada com outras atividades, tais como a bovinocultura. Tem-se observado a falta de técnicas de manejo voltadas para a reprodução e manejo sanitário para maximizar a eficiência do rebanho na produção, sendo a verminose nos rebanhos, a principal causa de perdas produtivas e econômicas (Silva et al., 2013).

A verminose é um dos principais problemas sanitários na ovinocultura, acarretando na redução no consumo voluntário de alimentos, afetando a digestão e absorção de nutrientes, na parte reprodutiva e produtiva do animal, interferindo também nas despesas adicionais como mão de obra, aquisição de medicamentos e mortalidade dos animais (Teixeira et al., 2015).

Dessa forma o manejo sanitário torna-se essencial para o desenvolvimento da atividade, uma vez que a principal causa das perdas produtivas e econômicas é a verminose, devido aos parasitas gastrointestinais, como por exemplo o Haemonchus contortus, parasita hematófago que se aloja no abomaso (Santana et al., 2016). Tem-se observado com maior frequência a resistência dos nematóides aos anti-helmínticos, deste modo gerando limitação no controle dos parasitas gastrointestinais (Kaplan et al., 2004).

Animais contaminados pelo Haemonchus contortus apresentam quadro anêmico, o que pode ser refletido e observado pela coloração da conjuntiva ocular relacionada com a carga parasitária presente no organismo do animal (Mugambi et al., 2005). Pensando em evitar o uso indiscriminado de anti-helmínticos e, consequentemente, a resistência parasitária, foram desenvolvidas técnicas que possibilitam identificar quais animais devem ser dosificados. O método FAMACHA tem como objetivo identificar animais com anemia causada pelo Haemonchus contortus (Molento et al., 2013a), avaliando a coloração da conjuntiva ocular.

Conciliando o método FAMACHA com a avaliação de Escore de Condição Corporal (ECC), que tem como objetivo avaliar o estado nutricional individual de cada animal e, através da mesma, saber quais medidas de manejo nutricional devem ser tomadas (Machado et al., 2008). 
A associação do método FAMACHA com o OPG (ovos por grama de fezes), é importante, pois o método identifica apenas parasitas hematófagos e o grau de anemia do animal. Já o OPG tem por finalidade identificar o grau de infecção do animal e quais parasitas além do $H$. contortus estão presentes no organismo do animal. Com a adoção desse manejo é possível reduzir os gastos com antiparasitários e consequentemente selecionar animais dentro rebanho em resistentes, resilientes e suscetíveis as cargas parasitárias (Minho \& Molento, 2014).

Dessa forma, este estudo teve como objetivo avaliar o perfil sanitário dos ovinos do IFRS - Campus Sertão, por meio da associação e aplicação do método FAMACHA e a avaliação do Escore de Condição Corporal (ECC) e, quando necessário, conduzir com o tratamento adequado.

\section{Metodologia}

As atividades foram desenvolvidas no Instituto Federal de Educação, Ciência e Tecnologia do Rio Grande do Sul Campus Sertão, no Setor de Zootecnia II, localizada no Distrito Engenheiro Luiz Englert, Rodovia RS 135, Km 25, município de Sertão, Rio Grande do Sul. O período do experimento foi do dia 26 de fevereiro de 2019 ao dia 11 de setembro de 2019.

O trabalho foi aprovado pela Comissão de Ética pelo Uso dos Animais - CEUA do IFRS, no Protocolo No 6345200219. A área disponível para a ovinocultura é de $50.000 \mathrm{~m}^{2}$. Na época o rebanho contava com 27 animais adultos e conforme foram nascendo os cordeiros o rebanho foi aumentando, somando no final das avaliações 53 animais. No entanto, para a condução desse experimento, considerou-se apenas o grupo inicial de 27 ovinos adultos. No mês de novembro foi realizada a venda de cordeiros machos, desta forma diminuindo o número de animais no rebanho. Também foi realizada a troca do reprodutor, como é feito anualmente, para evitar consanguinidade no lote. Para avaliação do Grau Famacha, utilizou-se a metodologia descrita por Fernandes et al. (2015a), e para avaliação do ECC, a metodologia experimental descrita por Fernandes et al. (2016b).

Os animais realizam pastejo durante o dia, com fornecimento de água ad libtum e farelo de milho (1\% do peso vivo) no cocho quando são recolhidos para o aprisco no final da tarde.

As avaliações sempre ocorriam uma vez por semana, sendo algumas avaliações no período matutino e outras no vespertino. Foram realizadas avaliações de ECC seguindo a escala de 1 a 5, sendo 1 o escore atribuído à um animal muito magro e 5 o escore atribuído à um animal muito gordo, de acordo com a metodologia descrita por Cezar e Souza (2007).

Simultaneamente à avaliação do ECC, era aplicado o método FAMACHA, observando a conjuntiva ocular dos animais para verificar o grau de anemia, de acordo com o cartão FAMACHA. Esse grau varia de 1 - vermelho vivo (indica animal saudável) ao 5 - branco pálido (animal anêmico), de acordo com a metodologia descrita por Van Wik e Bath (2002).

Os ovinos com ECC abaixo de 2 e grau FAMACHA 3, 4 ou 5 eram dosificados com o princípio ativo Monepantel 2,5\% $\left(\right.$ Zolvix $\left.^{\circledR}\right)$, na dose recomendada pelo fabricante. Em algumas avaliações também se realizava a coleta de fezes para OPG (ovos por gramas de fezes). As amostras eram coletadas diretamente da ampola retal e acondicionadas na própria luva que era utilizada para a retirada. Nesta era identificado o número do brinco do animal correspondente a cada amostra. A contagem de OPG era realizada seguindo a técnica descrita por Gordon e Withlock modificada por Ueno e Gonçalves (1998), para verificar a presença de parasitas no material coletado e conciliar com o ECC e o método FAMACHA.

Foram considerados para análise os dados dos 27 animais adultos durante 25 coletas realizadas semanalmente, entre março e setembro de 2019, totalizando 725 observações. Os dados foram tabulados e analisados por meio de distribuição de frequências e estatística descritiva.

\section{Resultados e Discussão}

No Gráfico 1, podemos observar que na maioria das avaliações os animais mantiveram o grau FAMACHA desejável, 
ou seja grau 1 (saudável). Também foi possível observar que nenhum animal apresentou o grau 5, ou seja, animal anêmico, mesmo diante de desafios de manejo, como por exemplo, épocas em que houve temperaturas amenas, dias chuvosos e pouca oferta de volumoso. Nestes períodos foi identificada a queda do ECC, no entanto os animais mantiveram o grau FAMACHA em níveis adequados.

Gráfico 1 - Grau FAMACHA de 27 ovinos adultos em 25 repetições.

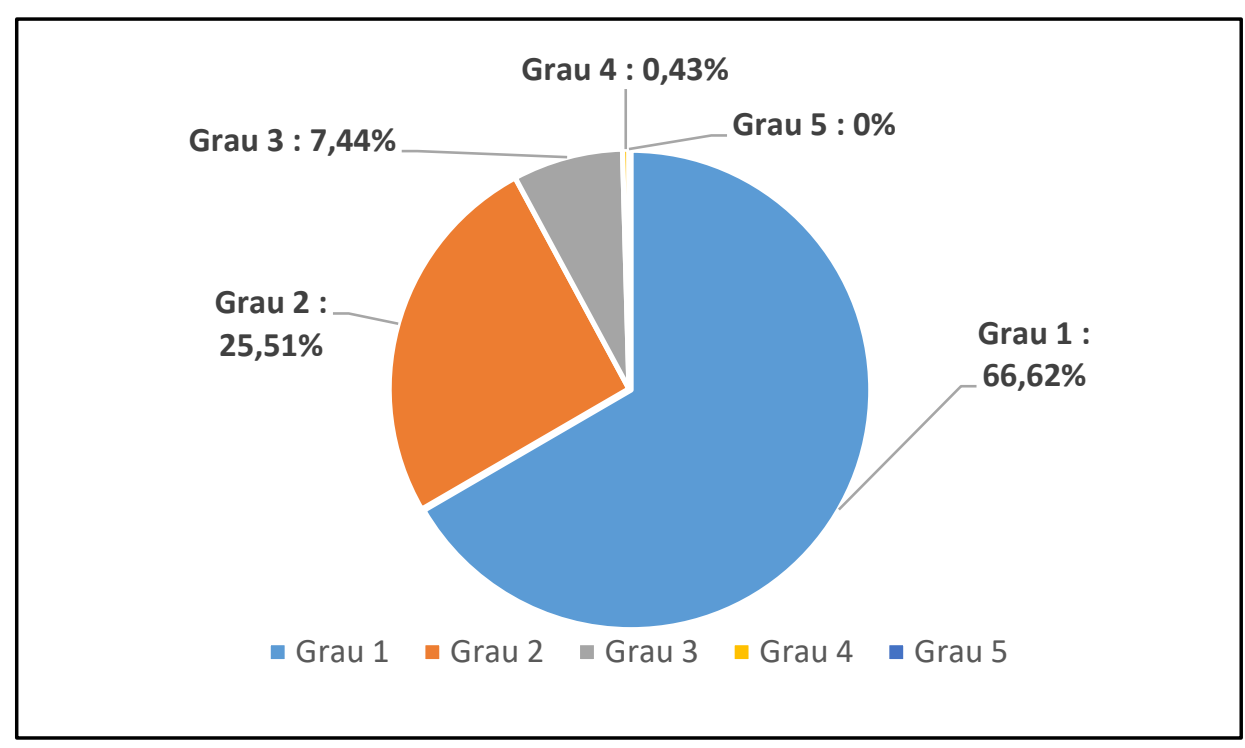

Fonte: Dados da pesquisa.

Para Amarante (2014b), a superlotação do rebanho nas pastagens contribui para uma maior contaminação das pastagens. O autor ressalta sobre a importância do estado nutricional do animal, que tem grande importância na eficiência da resposta imune e na capacidade de enfrentar/suportar as infecções parasitárias. Para que o organismo possa ter uma resposta imunológica adequada, deve-se atender as demandas por nutrientes que desempenham suas funções. Ou seja, além da disponibilidade de nutrientes na dieta, é necessário que sejam atendidas as necessidades fisiológicas do animal.

No Gráfico 2, observa-se o resultado relativo das avaliações de ECC realizadas no rebanho, no período em análise. Sendo importante observar que a maior parte das avaliações os animais se concentraram do ecores 2 ao 3,5. 
Gráfico 2 - Escore de Condição Corporal de 27 ovinos adultos em 25 repetições.

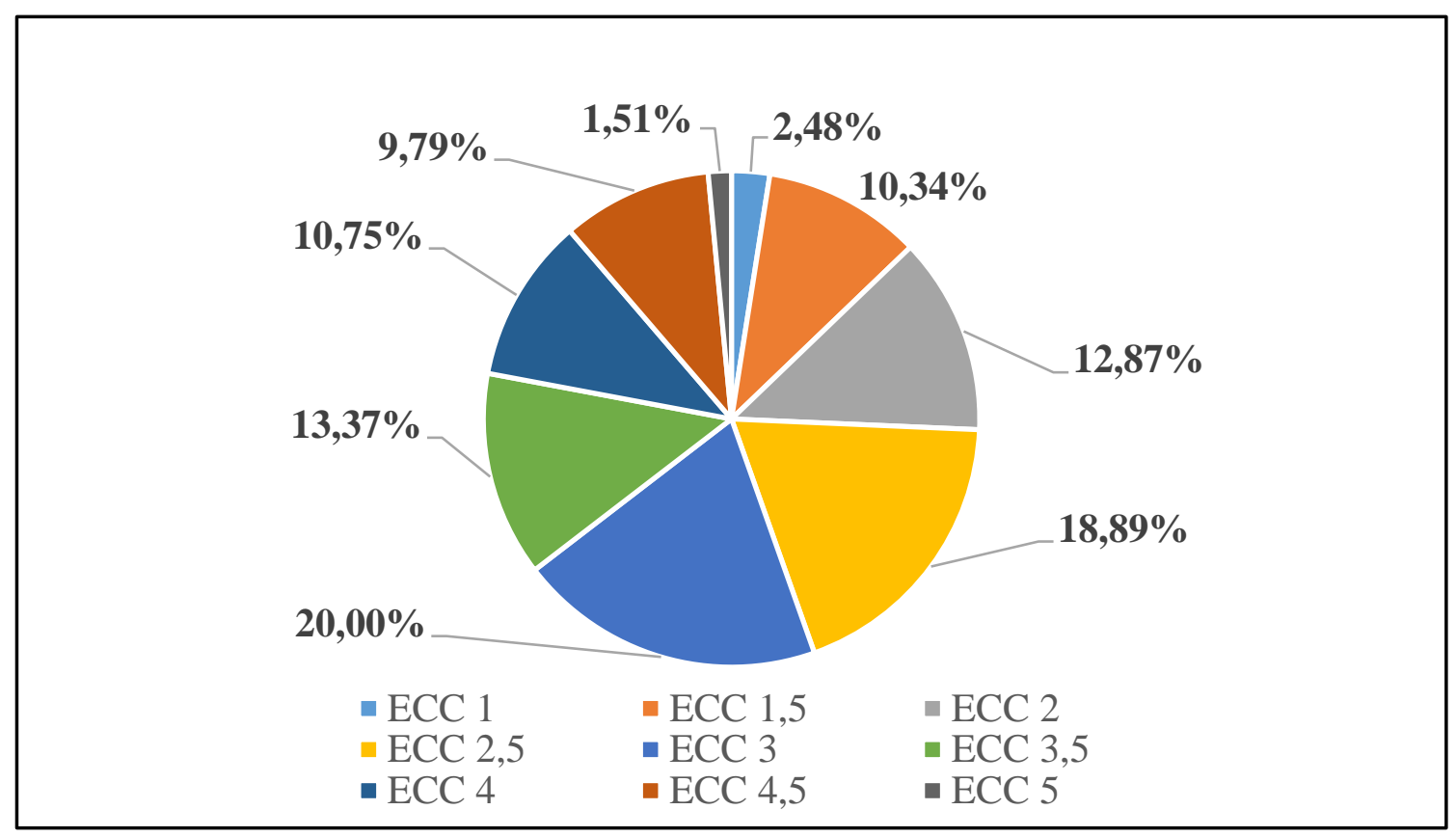

Fonte: Dados da pesquisa.

Quanto à necessidade de dosificações, das 725 avaliações apenas 3,72\% receberam vermífugo enquanto que 96,28\% possuíam carga parasitária baixa ou conseguiram suportar a carga parasitária sem apresentar sinais clínicos. Esse resultado pode ser justificado pelo fato dos animais serem adultos e consequentemente ter uma resposta imunológica mais eficiente, outra explicação para este acontecimento é a eficácia do princípio ativo usado para combater os parasitas presentes no rebanho, devido o seu uso adequado no lote.

Vilela et al. (2021), em 1356 avaliações em um rebanho ovino da raça Santa Inês do Nordeste brasileiro, relataram apenas 40 tratamentos com anti-helmínticos, correspondendo a 3\% das avaliações. Sendo 27 durante o período chuvoso e 13 durante o período seco. Neste rebanho, os autores relatam que a adoção do método FAMACHA promoveu a redução de $94 \%$ na administração de anti-helmínticos, quando comparados aos anos anteriores.

Molento et al. (2004b), em seu trabalho com ovinos adultos não puros naturalmente, num período de 180 dias, totalizando 45 dosificações de forma seletiva, observaram uma redução nas dosificações/custos em 75,6\%, quando comparado as dosificações no período anterior (185 doses), na propriedade.

O método FAMACHA pode proporcionar o uso seletivo de quimioterápicos nos animais e a redução nos gastos. Nas coletas realizadas pode-se observar que o método proporcionou a redução nos gastos com vermífugos em 46,3\%, quando comparado ao manejo tradicional com duas vermifugações estratégicas em todo o rebanho no período em análise.

Corroboram com os resultados encontrados, Cintra et al. (2020) que afirmam que o tratamento seletivo do rebanho resulta na economia nos custos com anti-helmínticos, além de proporcionar bem-estar ao animal e o controle na resistência parasitária.

Na Tabela 1 é possível observar que alguns animais precisaram ser dosificados mais de duas vezes, indicando que são suscetíveis a carga parasitária e apresentaram uma baixa eficiência na resposta imunológica, precisando de ajuda de quimioterápicos para poder combater as cargas parasitárias recorrentes em seu organismo. Também foi possível analisar que não demonstraram um grau de anemia muito alto, isso pode ser respondido pelo fato de ser detectado a tempo e contornar a situação o mais rápido possível. 
Tabela 1 - Animais que foram vermifugados.

\begin{tabular}{rccc}
\hline Brinco & Intervalo ECC & Intervalo FAMACHA & Semana da vermifugação \\
\hline 23 & $1 \mathrm{a} 3$ & $1 \mathrm{a} 3$ & $12^{\mathrm{a}}, 15^{\mathrm{a}}, 21^{\mathrm{a}}$ \\
53 & $1,5 \mathrm{a} 4$ & $1 \mathrm{a} 3$ & $9^{\mathrm{a}}, 11^{\mathrm{a}}, 15^{\mathrm{a}}, 21^{\mathrm{a}}$ \\
58 & $1,5 \mathrm{a} 4$ & $1 \mathrm{a} 3$ & $6^{\mathrm{a}}$ \\
60 & $1,5 \mathrm{a} 5$ & $1 \mathrm{a} 4$ & $8^{\mathrm{a}}, 10^{\mathrm{a}}, 12^{\mathrm{a}}, 15^{\mathrm{a}}, 21^{\mathrm{a}}$ \\
62 & $1 \mathrm{a} 3$ & $1 \mathrm{a} 3$ & $3^{\mathrm{a}}, 9^{\mathrm{a}}, 17^{\mathrm{a}}, 21^{\mathrm{a}}$ \\
64 & $2,5 \mathrm{a} 4$ & $1 \mathrm{a} 3$ & $2^{\mathrm{a}}$ \\
66 & $1 \mathrm{a} 4,5$ & $1 \mathrm{a} 4$ & $6^{\mathrm{a}}, 12^{\mathrm{a}}, 15^{\mathrm{a}}$ \\
68 & $2,5 \mathrm{a} 4,5$ & $1 \mathrm{a} 3$ & $3^{\mathrm{a}}, 12^{\mathrm{a}}$ \\
71 & $2,5 \mathrm{a} 4,5$ & $1 \mathrm{a} 3$ & $25^{\mathrm{a}}$ \\
73 & $2,5 \mathrm{a} 4,5$ & 1 & $6^{\mathrm{a}}$ \\
\hline
\end{tabular}

Fonte: Dados da pesquisa.

Na Tabela 2 foram selecionados animais que apresentaram grau FAMACHA igual ou inferior a 3, ECC maior ou igual a 2,5 e que durante as observações não necessitaram de vermifugações, indicando a ideia de animais resistentes e resilientes.

Tabela 2 - Frequência de animais com ECC acima de 2,5 e grau FAMACHA inferior a 3.

\begin{tabular}{cccccccccc}
\hline \multirow{2}{*}{ Brinco } & \multicolumn{3}{c}{ ECC } & \multicolumn{5}{c}{ GRAU FAMACHA } \\
\cline { 2 - 9 } & $\mathbf{2 , 5}$ & $\mathbf{3}$ & $\mathbf{3 , 5}$ & $\mathbf{4}$ & $\mathbf{4 , 5}$ & $\mathbf{5}$ & $\mathbf{1}$ & $\mathbf{2}$ & $\mathbf{3}$ \\
\hline Carneiro & & 2 & 5 & 10 & 8 & & 25 & & \\
$558 \mathrm{~A}$ & & & & 1 & 13 & 11 & 20 & 4 & 1 \\
55 & & 10 & 8 & 4 & 3 & & 16 & 8 & 1 \\
64 & 12 & 6 & 6 & 1 & & & 13 & 10 & 2 \\
67 & 1 & 6 & 7 & 5 & 6 & & 9 & 13 & 3 \\
68 & 1 & 12 & 5 & 4 & 3 & & 4 & 16 & 5 \\
70 & & & 2 & 15 & 8 & & 25 & & \\
71 & 2 & 1 & 2 & 4 & 16 & 23 & 1 & 1 \\
73 & 7 & 6 & 10 & 1 & 1 & & 25 & & \\
\hline
\end{tabular}

Fonte: Dados da pesquisa.

Oliveira et al. (2011), observaram que a correlação entre o método FAMACHA é proporcional ao OPG, pois quanto maior o grau FAMACHA maior será a contagem de ovos. A correlação entre o volume globular com o método são inversamente proporcionais, quanto maior for o volume globular menor grau FAMACHA o animal apresenta. Os autores ressaltaram que é importante conciliar o método FAMACHA com OPG e coprocultura para encontrar outros parasitas no organismo do animal.

A partir da contagem de OPG, os animais puderam ser classificados com maior critério através da constatação da carga parasitária após a vermifugação, verificando assim a eficácia do tratamento e a correlação da carga parasitária e a resposta do organismo com o grau FAMACHA.

A classificação dos animais foi baseado nos critérios de Amarante (2014b) e Storillo (2016), onde animais avaliados através da contagem de OPG que demonstraram redução na contagem, foram classificados como resistentes, os classificados como resilientes, apresentaram uma contagem acima de 900 parasitas adultos e os suscetíveis de 2.000 a 3.000 parasitas.

Dessa maneira, os animais foram classificados em resistente, resilientes e suscetíveis, conforme a Tabela 3. Baseandose nesses critérios, foram encontrados 10 animais resistentes (35,71\%), 12 resilientes (42,86\%) e seis animais suscetíveis $(21,43 \%)$. 
Tabela 3 - Classificação dos animais de acordo com a associação entre avaliação de ECC, grau FAMACHA e contagem de OPG.

\begin{tabular}{|c|c|c|c|}
\hline Brinco & Resistente & Resiliente & Suscetível \\
\hline Carneiro & $\mathrm{X}$ & & \\
\hline 558 & $\mathrm{X}$ & & \\
\hline 23 & & & $X$ \\
\hline 51 & & $\mathrm{X}$ & \\
\hline 52 & & $X$ & \\
\hline 53 & & & $X$ \\
\hline 55 & $X$ & & \\
\hline 56 & & $X$ & \\
\hline 57 & $X$ & & \\
\hline 58 & & $X$ & \\
\hline 59 & & $X$ & \\
\hline 60 & & & $X$ \\
\hline 61 & & $\mathrm{X}$ & \\
\hline+2 & & & $\mathrm{X}$ \\
\hline 63 & & $X$ & \\
\hline 64 & & $X$ & \\
\hline 65 & $\mathrm{X}$ & & \\
\hline 66 & & & $X$ \\
\hline 67 & & $\mathrm{X}$ & \\
\hline 68 & & $X$ & \\
\hline 69 & & $X$ & \\
\hline 70 & $X$ & & \\
\hline 71 & $X$ & & \\
\hline 72 & $X$ & & \\
\hline 73 & & $X$ & \\
\hline 74 & $X$ & & \\
\hline 75 & $X$ & & \\
\hline
\end{tabular}

Fonte: Dados da pesquisa.

Para a classificação de animais resilientes, os animais devem suportar altas cargas parasitárias com baixo comprometimento na produtividade (Albers et al., 1987). Os animais jovens são suscetíveis, podendo desenvolver imunidade e, apresentar na idade adulta o mecanismo de resistência às infecções (Sotomaior et al., 2007). Mas os animais sensíveis/suscetíveis apresentam alta carga parasitária acompanhada com baixos valores de hematócrito.

Os animais que foram dosificados e diminuíram a contagem e que não precisaram mais de tratamento foram classificados como resilientes. Já os animais que demandam maior número de vermifugações, eram classificados como suscetíveis. Estes animais devem ser descartados do rebanho pois aumentam a incidência de parasitos no rebanho (Storillo, 2016).

\section{Considerações Finais}

Os resultados demonstram a importância da adoção de um manejo integrado na ovinocultura e os benefícios produtivos obtidos a partir do controle zootécnico do rebanho, buscando proporcionar melhor desempenho produtivo e sanitário, além de evitar o uso indiscriminado de antihelmínticos e aprimorar as estratégias de seleção dos animais. Se fazendo importante a adoção nas propriedades, pois foi possível constatar a eficiência do controle sanitário no rebanho. 
Ainda evidencia a importância de se ter um planejamento forrageiro, onde se possa fornecer pastagem durante o ano todo, junto com uma suplementação, afim de suprir nutricionalmente o que os animais não conseguem retirar das plantas forrageiras. A partir de um manejo nutricional adequado, espera-se obter bom desempenho produtivo e reprodutivo, sendo imprescindível conhecer as exigências nutricionais dos animais.

Sendo assim, estudos futuros devem ser realizados para verificar a correlação entre a aplicação do método FAMACHA e a avaliação do Escore de Condição Corporal (ECC) em diferentes categorias de ovinos e em ambientes produtivos distintos.

\section{Agradecimento}

Ao Instituto Federal do Rio Grande do Sul (IFRS) pela concessão de bolsa de iniciação científica (Fomento Interno) às duas primeiras autoras.

\section{Referências}

Alberts, G. A .A., Grey, G. D., Piper, L. R.., Barker, J. S. F., Jambre, J. F. Le., \&, Barger, I. A. (1987). The Genetic Resistance and Resiliense to Haemonchus contortus Infection in Young Merino Sheep. International Journal for Parasitology, 17(1), 1355-1363.

Amarante, A. F. T.(2014). Classe nematoda. In: Os parasitas de ovinos, Editora UNESP, 13-97.

Arco (2021). Associação Brasileira de Criadores de Ovinos. Padrões raciais (2021). Bagé- RS.http://www.arcoovinos.com.br/index.php/mn-srgo/mnpadroesraciais/41-suffolk.

Cezar, M. F., \& SousA, W. H.(2007). Carcaças ovinas e caprinas: obtenção - avaliação - classificação. Agropecuária tropical. 232.

Cintra, M. C. R.,, Vieira, D. L., Rezende., E. A., \& Molento, M. B. (2020). Avaliação econômica da utilização do tratamento seletivo nas infecções parasitárias em ovinos. Archives of Veterinary Science. 25(5) Esp. I Semana Acadêmica da Pós - Graduação em Ciências Veterinárias - UFPR (I SAPGCV), 110.

Fernandes, M. A. M., Gilaverte, S., Buzatti, A., Sprenger, L. k., Silva, C. J. A., Peres, M. T. P., Molento, M. B., \& Monteiro, A. L. G. (2015a). Método FAMACHA para detectar anemia clínica causada por Haemonchus contortus em cordeiros lactentes e ovelhas em lactação. Pesq. Vet. Bras. 35(6):525530, 10.1590/S0100-736X2015000600006.

Fernandes, A. F. A., Oliveira, J. A., \& Queiroz, S. A. (2016b). Escore de condição corporal em ruminantes. ARS Veterinária. 32(11), 055-066.

Gordon, H. M. C. L., \& Whitlock, A. V. (1939). A new technique for counting nematode eggs in sheep feces. Journal Council Scientific Industry Research Australia, 12, 50-52.

Kaplan, R. M., Burke, J. M., Terril, T. H., Miller., J. E., Getz, W. R.., Mobini, S., Valencia, E., Williams, M. J., Williamson, L. H., Larsen, M., \& Vatta, A. F. (2004). Validation of the FAMACHA® eye color chart for detecting clinical anemia in sheep and goats on farms in southern United States. Veterinary Parasitology, 123(1), 105-120.

Oliveira, M. V., Moura, M. S., \& Barbosa, F. C. (2011). Avaliação comparativa do método FAMACHA®, volume globular e OPG em ovinos. PUBVET. 5(7), 154, Art. 1039.

Machado, R., Correa, R. F., Barbosa, R. T., \& Bergamaschi, M. A. C. M.(2008). Escore de condição corporal e sua aplicação no manejo reprodutivo de ruminantes. Embrapa Pecuária Sudeste - Circular Técnica 57.

Minho, A. P., \& Molento, M. B. (2014). Método FAMACHA: uma técnica para prevenir o aparecimento da resistência parasitária. Revista Embrapa Pecuária Sul - Circular Técnica 46

Molento, M. B., Veríssimo, C. J., Amarante, A. T., Van Wyk, J. A., Chagas, A. C. S., Araújo, J. V., \& Borges, F. A. (2013). Alternativas para o controle de nematoides gastrintestinais de pequenos ruminates. Arquivos do Instituto Biológico. 80(2), 253-263.

Molento, M. B., Tasca, C., Gallo, A., Ferreira, M., Bononi, R.., \& Stecca, E. Método FAMACHA como parâmetro clínico individual de infecção por Haemonchus contortuts em pequenos ruminantes. Ciência Rural. 34(4).

Mugambi, J. M., Audho, J. O., Njomo, S., \& Baker, R. L. (2005). Evaluation of the phenotypic performance of a Red Massai and Dorper double backcross resource population: indoor trickle challenge with Haemonchus contortus. Veterinary Parasitology, 127: 263-275.

Santana, T. M., Dias, F. J., Santello, G. A., Lopes, M., Melo, T. T., Pantoja, M. C., \& Almeida, L. M. A. (2016). Utilização de métodos auxiliares na identificação endoparasitária em ovelhas no Amazonas. Revista Brasileira de Higiene e Sanidade Animal, 10(3), 436 - 446.

Silva, A. P. S. P., Santos, D. V., JR. Kohek, I., Machado, G., Hein, H. E., Vidor, A. C. M., \& Coberllini, L. G. (2013). Ovinocultura do Rio Grande do Sul: descrição do sistema e dos principais aspectos sanitários e reprodutivos. Pesquisa Veterinária Brasileira. 33(12), $1453-1458$.

Sotomaior, C. S., De Carli, L. M., Tangleica, L., Kaiber, B. K., \& Souza, F. P. (2007). Identificação de ovinos e caprinos resistentes e susceptíveis aos helmintos gastrintestinais. Revista Acadêmica - Ciências Agrárias e Ambientais, 5(4), 397 - 412.

Storillo, V. M. (2016). Resistência, resiliência e sensibilidade de ovinos a Haemonchus contortus: comparações hematológicas e bioquímicas. Tese de 
Research, Society and Development, v. 11, n.2, e40311225860, 2022

(CC BY 4.0) | ISSN 2525-3409 | DOI: http://dx.doi.org/10.33448/rsd-v11i2.25860

Doutorado. USP.

Teixeira, M., Cavalcante, A. C. R., \& Vieira, L. S. (2015). Controle de verminoses em caprinos e ovinos. Embrapa-Caprinos e Ovinos. Sobral, CE.

Ueno, H., \& Gonçalves, P. C. (1998). Manual para diagnóstico das helmintoses de ruminantes. (4a ed.), Tokyo: Japan International Cooperation Agency. 143 p.

Van Wyk, J. A., \& Bath, G. F. (2002). The FAMACHA system for managing haemonchosis in sheep and goats by clinically identifying individual animals for treatment. Veterinary Research, 33, 509-529.

Vilela, V. L. R., Bezerra, H. M. F. F., Bezerra, R. A., Dantas, M. O., Alcântara, E. T., Oliveira, L. V. S., Nóbrega, K. S., Calazans, F. B., Feitosa, T. F., Braga, F. R., \& Molento, M. B. (2021). Sustainable agriculture: the use of FAMACHA method in Santa Ines sheep in the Semi-arid region of Brazil. Semina, 42(3), 1647-1662. 